\title{
Results of the IRAM M33 Large Program
}

\author{
Jonathan Braine $^{1}$, Karl Schuster ${ }^{2}$, Pierre Gratier ${ }^{2}$ and \\ Clément Druard ${ }^{1,2}$ \\ ${ }^{1}$ Univ. Bordeaux, Laboratoire d'Astrophysique de Bordeaux, CNRS, LAB, UMR 5804, \\ F-33270, Floirac, France. \\ email: braine@obs.u-bordeaux1.fr \\ ${ }^{2}$ IRAM, 300 Rue de la Piscine, F-38406 St Martin d'Hères, France
}

\begin{abstract}
A very sensitive survey of the $\mathrm{CO}(2-1)$ emission of the entire disk of the Local Group galaxy M 33 is virtually complete. Hundreds of clouds down to a mass of $10000 \mathrm{M}_{\odot}$ are detected and we show that the cloud mass function (assuming a constant ratio between CO emission and $\mathrm{H}_{2}$ mass) steepens away from the center of $\mathrm{M} 33$. Through comparison with dust emission and cloud Virial masses, we estimate a $N\left(\mathrm{H}_{2}\right) / I_{\mathrm{CO}}$ factor roughly twice Galactic.
\end{abstract}

Keywords. galaxies: individual (M33) - Local Group — galaxies: evolution — galaxies: ISM — ISM: clouds — stars: formation

\section{Why observe M 33?}

The Local Group galaxy Messier 33 is very nearby ( $840 \mathrm{kpc}$, where 1" = 4pc), such that individual molecular clouds can be resolved. The Local Group contains only three true spiral galaxies (M31, M33, and the Milky Way) and M 33 is the only one which is not seen edge-on. The inclination of $\mathrm{M} 33,56^{\circ}$, is optimal, yielding a clear view of the dynamics of the disk with a clean line of sight. M 33 has an interstellar radiation field similar to spirals like our own.

Nonetheless, there are important differences which make M 33 a younger object than the Milky Way or M 31. M 33 is bluer and more gas-rich than the Milky Way and it is smaller, less massive by roughly a factor 10, and has a lower metallicity by roughly a factor two. While M 33 is certainly not a typical early universe object, these features make it an ideal stepping-stone towards lower metallicity objects to understand the ISM and star formation in primitive environments. Because it is only marginally different from large local spirals like our own, we can use standard techniques to study the interstellar medium (ISM) and how it is affected by variations in metallicity.

Using the 30meter radiotelescope of the Institut de RadioAstronomie Millimétrique (IRAM), we have carried out a Large Program covering the entire disk of M 33 in the $\mathrm{CO}(J=2-1)$ line at $230 \mathrm{GHz}$. Among the goals are $(i)$ the identification of the Giant Molecular Clouds (GMCs) in M 33, (ii) their lifetime and link with star formation, (iii) a statistical measure of the average $N\left(\mathrm{H}_{2}\right) / I_{\mathrm{CO}}$ ratio, $(i v)$ the GMC mass function, $(v)$ the detection of a size-linewidth relation for M33, and $(v i)$ the link between the structures seen in $\mathrm{CO}$ and so-called "Dark $\mathrm{H}_{2}$ ". Detailed results can be found in Gardan et al. (2007); Gratier et al. (2010, 2012) and Druard et al. (in prep.).

\section{Observations and Analysis}

M 33 was observed with the 9 pixel dual polarization receiver HERA in the On-TheFly mode (Gardan et al. 2007; Gratier et al. 2010). After eliminating poor data, spectra were combined into a datacube with a spectral resolution of $2.6 \mathrm{~km} / \mathrm{s}$ and 12 " spatial 
resolution. Figure 1 shows the integrated intensity map (left) and the noise map (right panel).

The cloud-finding algorithm CPROPS (Rosolowsky \& Leroy 2006) was then used on the entire cube to identify coherent clouds. Applied to the 2010 dataset, a cloud sample of 337 clouds was defined (Gratier et al. 2012), then and still the largest extragalactic sample of GMCs. In order to estimate the durations of the different stages of a GMCs "life" (i.e. the time it is recognizable as a GMC), the clouds were divided into three types based on the emission in the FUV, $\mathrm{H} \alpha, 8 \mu \mathrm{m}$, and $24 \mu \mathrm{m}$ bands within the cloud contours. Observations in these bands all have better spatial resolution than the 12" resolution of the CO. GMCs without star formation (type A) were fairly straightforward to identify; the distinction between types B and C (embedded vs. exposed star formation), was based on the strengths in the FUV and $\mathrm{H} \alpha$ bands, which trace exposed star formation (see details in Gratier et al. 2012). The cloud types are not unlike those of Kawamura et al. (2009) but the classification was done quite differently. As the flux distributions were monomodal (single bump in histogram of cloud intensities), choosing a cutoff flux to define a category would result in $(a)$ the number of clouds depending very sensitively on the value of the cutoff flux and $(b)$ a sharp radial increase in GMCs without star formation (or with only embedded SF) because of the decrease in cloud fluxes in all bands with galactocentric distance.

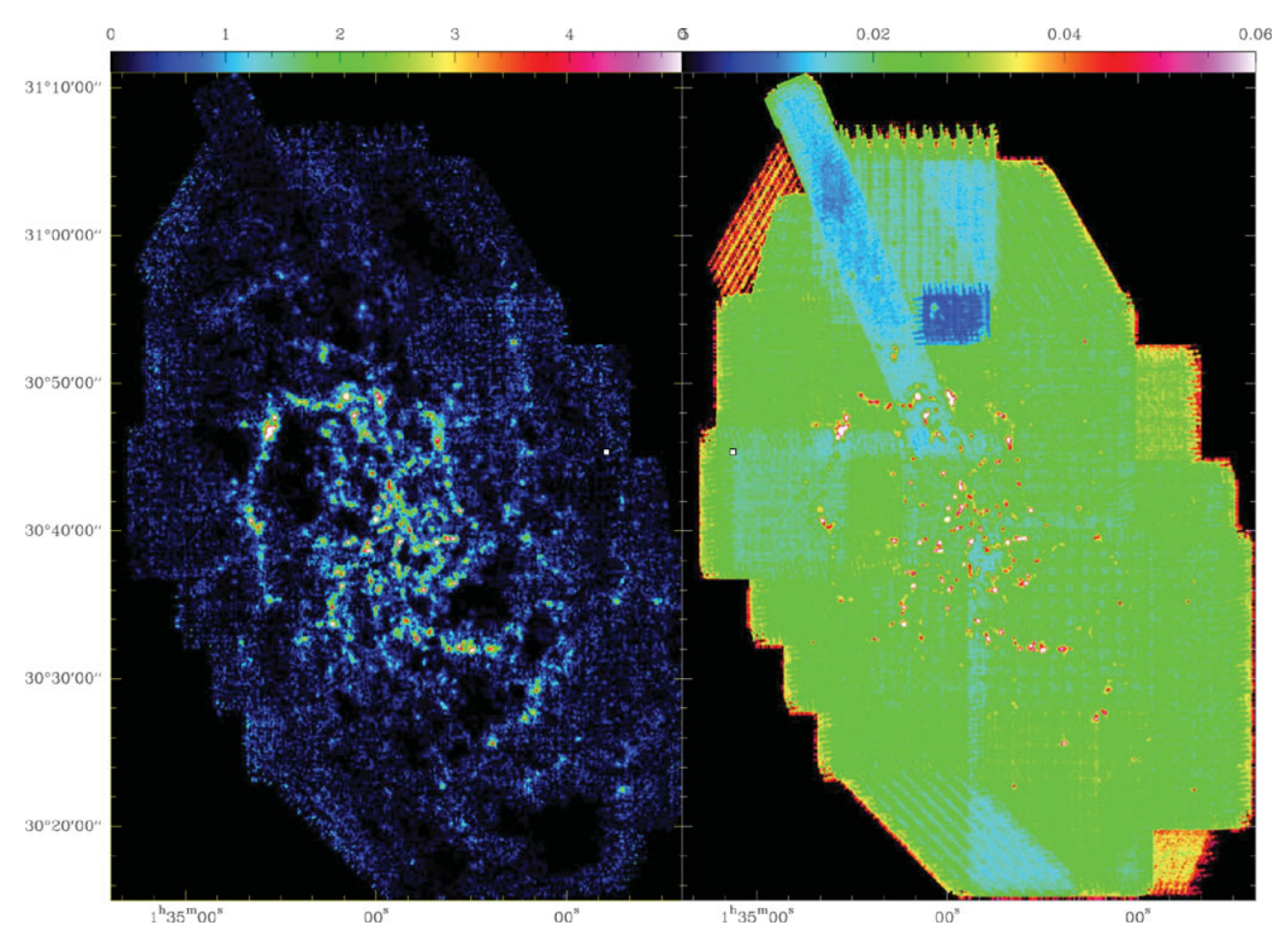

Figure 1. Results of $\mathrm{CO}$ observations of $\mathrm{M}$ 33: left panel shows the integrated intensity in $\mathrm{K} \mathrm{km} \mathrm{s}^{-1}$ while the right panel shows the noise in Kelvins. We have not excluded the regions with $\mathrm{CO}$ signal from the noise calculation so bright clouds are readily seen in the noise map. 


\section{Results and Conclusions}

We estimate that $1 / 6$ of the 337 GMCs are without star formation, reasonably consistent with the 1/4 found by Kawamura et al. (2009) for the LMC. It is worth noting that this is really a minimum because there could be emission along the line of sight to the cloud, but not related to the cloud, causing it to be classified B or C. From these observations, it seems that GMCs spend about $20 \%$ of their duration before massive star formation commences. Using the Herschel data on M 33 (Kramer et al. 2010), it was possible to show that the dust temperatures of the type A clouds were on average about $1.5 \mathrm{~K}$ lower than in clouds showing star formation.

In our sample there was no significant difference in size or spatial distribution between the type A clouds and those with star formation. However, the GMCs without star formation had lower CO line temperatures and intensities. For all classes, the CO became weaker with galactocentric distance and the $\mathrm{CO}$ intensity was linked to the presence of strong HI emission. It is interesting that the Larson (1981) size-linewidth relation is not found at the resolution of our data.

The cloud mass spectrum is a major question is astrophysics, especially since at small scales it is expected to be related to the stellar IMF, but also because we would like to know whether most of the molecular gas is in large or small clouds. Going from the observed CO luminosities to cloud masses requires either a conversion factor between $\mathrm{CO}$ luminosity and $\mathrm{H}_{2}$ mass or the assumption that cloud mass is traced by cloud size and linewidth. Our sample covers nearly 2 orders of magnitude in cloud CO luminosities with more clouds than used previously. If $\mathrm{CO}$ luminosity is a tracer of $\mathrm{H}_{2}$ mass, the assumption made by all previous authors, then we find that $n(m) \propto m^{-2}$ when all clouds are included but that the inner disk spectrum is flatter $\left(n(m) \propto m^{-1.6}\right)$ and outer disk steeper $\left(n(m) \propto m^{-2.3}\right)$, such that most of the mass should be in massive GMCs in the inner parts but in smaller clouds further out in the disk (see details in Gratier et al. 2012).

M 33 has a subsolar metallicity and this is expected to raise the $N\left(\mathrm{H}_{2}\right) / I_{\mathrm{CO}}$ conversion factor with respect to the Galactic average due essentially to the deeper photodissociation of CO in the GMC envelopes of low-metallicity clouds. As dust and gas are thoroughly mixed, it is possible to use measurements of the dust emission to constrain the amount of gas present. While this is not straightforward, Braine et al. (2010b) estimate an average $N\left(\mathrm{H}_{2}\right) / I_{\mathrm{CO}} \approx 4 \times 10^{20} \mathrm{~cm}^{-2} /\left(\mathrm{K} \mathrm{km} \mathrm{s}^{-1}\right)$, twice the usual Galactic value, with a radial variation such that the amount of $\mathrm{CO}$ emission per unit molecular gas is higher in the inner galaxy.

\section{A detailed view of an outer galaxy GMC}

Very few molecular clouds have been observed far from galactic centers and this is particularly true for low-metallicity systems. The molecular cloud M33-18, first observed by Braine et al. (2010a), is at the edge of the optical disk of M 33 and was re-observed by Braine et al. (submitted) in the $\mathrm{CO}(1-0)$ line at high spatial resolution with the IRAM Plateau de Bure interferometer. The cloud is part of a distant spiral arm and the orientation of the molecular cloud follows that of the HI arm. At such large distances from the center of M 33, the stellar disk has a very low surface density such that the mass is dominated by the $\mathrm{HI}$ and $\mathrm{H}_{2}$ gas. Some emission in the FUV and $24 \mu m$ bands is present and we estimate a star formation rate of about $4 \mathrm{M}_{\odot} / \mathrm{Myr}$ from the $24 \mu \mathrm{m}$ flux.

The high resolution $\mathrm{CO}(1-0)$ observations reveal that the cloud is a single entity rather than a series of distinct smaller clouds. The line width is narrow, about $2.5 \mathrm{~km} \mathrm{~s}^{-1}$, at all spatial resolutions available, which range from $10 \mathrm{pc}$ to the whole cloud. Using the same 


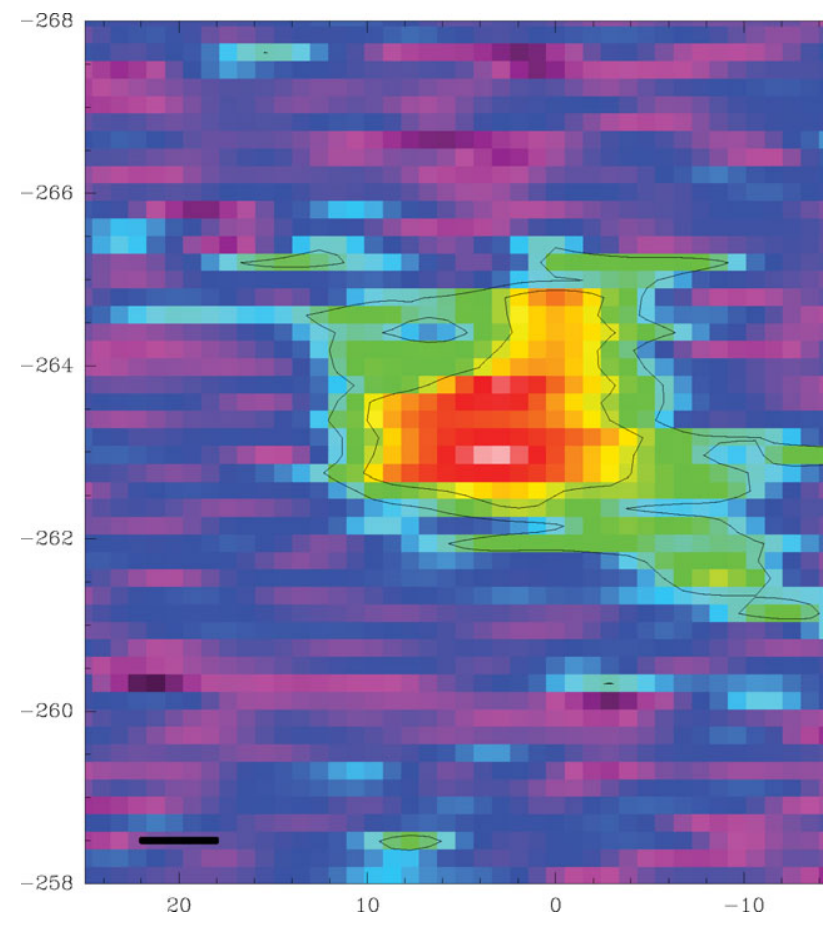

Figure 2. PV-diagram of the outer disk cloud along a cut with a position angle of $49^{\circ}$ East of North. The Y-axis indicates recession velocity in $\mathrm{km} \mathrm{s}^{-1}$ from slower rotation $\left(-258 \mathrm{~km} \mathrm{~s}^{-1}\right)$ to faster rotation velocities. The $\mathrm{X}$-axis indicates the distance from the cloud center in arcseconds, with negative values being to the south-west. Contours at 0.06 and $0.12 \mathrm{Jy} / \mathrm{beam}$ are shown. The bar to the lower left indicates 4", as the beam measures $4.50 \times 3.49$ ".

techniques as for Galactic clouds, we estimate Virial masses and deduce a CO-to- $\mathrm{H}_{2}$ conversion ratio of $N\left(\mathrm{H}_{2}\right) / I_{\mathrm{CO}(1-0)}=4 \pm 0.5 \times 10^{20} \mathrm{~cm}^{-2} /\left(\mathrm{K} \mathrm{km} \mathrm{s}^{-1}\right)$.

While the velocity of the CO-bright regions is very constant, a low-brightness region to the SW is present with a velocity $\left(-261 \mathrm{~km} \mathrm{~s}^{-1}\right)$ such that it is approaching the main body of the cloud. This region can be seen to the lower right of the position-velocity diagram shown in Fig. 2. A more marginal feature can be seen to the NE (upper left) at about $-265 \mathrm{~km} \mathrm{~s}^{-1}$, which also corresponds to material approaching the cloud. If this gas is falling onto the bright part of the cloud, the accretion rate is about $0.0002 \mathrm{M}_{\odot} / \mathrm{yr}$.

\section{References}

Braine, J., Gratier, P., Kramer, C., et al. 2010a, A\&A, 520, A107+ Braine, J., Gratier, P., Kramer, C., et al. 2010b, A\&A, 518, L69+

Gardan, E., Braine, J., Schuster, K. F., Brouillet, N., \& Sievers, A. 2007, A $6 A, 473,91$

Gratier, P., Braine, J., Rodriguez-Fernandez, N. J., et al. 2012, A\&GA, 542, A108

Gratier, P., Braine, J., Rodriguez-Fernandez, N. J., et al. 2010, A\& A, 522, A3

Kawamura, A., Mizuno, Y., Minamidani, T., et al. 2009, ApJS, 184, 1

Kramer, C., Buchbender, C., Xilouris, E. M., et al. 2010, A\&A, 518, L67

Larson, R. B. 1981, MNRAS, 194, 809

Rosolowsky, E. \& Leroy, A. 2006, PASP, 118, 590 